\title{
Improved Riemann Ladder Function
}

\author{
Dan Liu \\ Sichuan Normal University, Chengdu, China \\ Email: 576672568@qq.com
}

How to cite this paper: Liu, D. (2021) Improved Riemann Ladder Function. Journal of Applied Mathematics and Physics, 9, 584-593.

https://doi.org/10.4236/jamp.2021.94042

Received: March 5, 2021

Accepted: April 13, 2021

Published: April 16, 2021

Copyright () 2021 by author(s) and Scientific Research Publishing Inc. This work is licensed under the Creative Commons Attribution International License (CC BY 4.0).

http://creativecommons.org/licenses/by/4.0/

\section{(c) (i) Open Access}

\begin{abstract}
In Riemann's prime distribution formula, there is a key Riemann ladder function. This is the basis for Riemann to study the distribution of prime numbers. But the calculation of Riemann's ladder function is very complicated. According to the definition of Riemannian ladder function, we greatly improve the Riemannian ladder function, obtain new ladder function and improved Riemannian prime distribution formula, and prove the improved Riemannian prime distribution formula. We use the improved Riemannian prime distribution formula and merdens theorem to obtain a strong prime theorem.
\end{abstract}

\section{Keywords}

Ladder Function, Prime Distribution, Metens Theorem, Strong Prime Theorem

\section{Introduction}

The distribution of prime numbers is a difficult problem in number theory. The proof of prime theorem attracts many scholars and puzzles many wise people. Mathematicians have proved thousands of theorems on the premise of Riemann conjecture.

In 1737, the Swiss mathematician Leonhard Euler published a formula:

$$
\sum_{n=1}^{\infty} n^{-s}=\prod_{p}\left(1-p^{-s}\right)^{-1}, s>1,
$$

Here (1.1) is called Euler product formula. Where $n$ is an integer, $p$ is a prime and $s$ is a real number.

Euler studied this formula. Let $s=1$. For large $x$, Euler obtains an asymptotic formula

$$
\sum_{p \leq x} \frac{1}{p} \sim \log \log x
$$


From 1792 to 1793, Gauss, a German mathematician, studied the number of primes in 1000 adjacent integers around $x$. he found that for large $x$, the "average distribution density" of primes should be $1 / \log x$, which is shown as follows [1] [2] [3] [4] [5]:

$$
\frac{\pi(x)-\pi(x-1000)}{1000} \sim \frac{1}{\log x}
$$

where, the number of primes not exceeding $x$ is $\pi(x)$. General expression

$$
\frac{\pi(x)-\pi(x-r)}{r} \sim \frac{1}{\log x}, r \ll x
$$

Here (1.3) is Gauss's guess. Represents the number of primes in $r$ adjacent integers near $x$.

\section{For example}

Let $x=1238, r=238$, by (1.3) get

$$
\frac{\pi(1238)-\pi(1238-238)}{238}=\frac{203-168}{238}=0.14705 \sim \frac{1}{\log 1238}=0.14042
$$

According to (1.3), we can divide $x$ into $n$ parts. In this way, we can get $\pi(x)$. Let integern, $x=n r$, by (1.3) can get

$$
\pi(n r)-\pi(n r-r) \sim \frac{r}{\log (n r)}
$$

Set up $n=1,2,3, \cdots, a$, Substituting (1.4) get

$$
\begin{gathered}
n=1, \pi(r)-\pi(0) \sim \frac{r}{\log (r)}, \\
n=2, \pi(2 r)-\pi(r) \sim \frac{r}{\log (2 r)}, \\
n=3, \pi(3 r)-\pi(2 r) \sim \frac{r}{\log (3 r)}, \\
\vdots \\
n=a, \pi(a r)-\pi(a r-r) \sim \frac{r}{\log (a r)}
\end{gathered}
$$

Add each

$$
\begin{aligned}
& \pi(r)-\pi(0)+\pi(2 r)-\pi(r)+\pi(3 r)-\pi(2 r)+\cdots+\pi(a r)-\pi(a r-r) \\
& \pi(a r)=\frac{r}{\log (r)}+\frac{r}{\log (2 r)}+\frac{r}{\log (3 r)}+\cdots+\frac{r}{\log (a r)},
\end{aligned}
$$

Let $x=a r$, get

$$
\pi(x) \sim r \sum_{n=1}^{a} \frac{1}{\log (n r)}, a=\frac{x}{r}
$$

Here (1.5) is a prime theorem without remainder. It is equal to the prime theorem $\pi(x) \sim \operatorname{Lix}$.

For example 
Let $x=64, r=16, a=x / r=4$, by (1.6) calculation,

$$
\pi(x) \sim 16\left(\frac{1}{\log 16}+\frac{1}{\log 32}+\frac{1}{\log 48}+\frac{1}{\log 64}\right) \sim 18,
$$

actual $\pi(64)=18$.

In this paper, we improve the Riemann ladder function and prove a strong prime number theorem by using the metens theorem [3].

$$
\begin{gathered}
\pi(x)=s(x)+O\left(x^{1 / 2} \log x\right),(x \rightarrow \infty), \\
s(x)=(x+1) \log \log x-2 \sum_{n=1}^{a} \log \log (2 n), a=x / 2,
\end{gathered}
$$

Before proving (1.6), we use a simple method to prove (1.3). Obviously, if we prove (1.3), we will prove (1.5).

\section{The Elementary Proof of Prime Number Theorem}

It is difficult to prove the prime theorem. It is especially difficult to prove the prime theorem in a simple way. We prove the prime theorem (1.3) by using the simple method according to Euler's asymptotic formula

Prove

Let the integer $r$, prime $p, x-r \leq p \leq x$ obviously [6]

$$
(x-r) \sum_{x-r \leq p \leq x} \frac{1}{p} \leq \pi(x)-\pi(x-r) \leq x \sum_{x-r \leq p \leq x} \frac{1}{p},
$$

\section{For example}

Let $x=16, r=12, x-r=4,4 \leq p \leq 16, \pi(16)-\pi(4)=4$, by (2.1) get

$$
4\left(\frac{1}{5}+\frac{1}{7}+\frac{1}{11}+\frac{1}{13}\right)<\pi(16)-\pi(4)<16\left(\frac{1}{5}+\frac{1}{7}+\frac{1}{11}+\frac{1}{13}\right),
$$

By (2.1) can ge

$$
x(1-r / x) \sum_{x-r \leq p \leq x} \frac{1}{p} \leq \pi(x)-\pi(x-r) \leq x \sum_{x-r \leq p \leq x} \frac{1}{p},
$$

Let $r=x^{1 / 2}$, get

$$
\lim _{x \rightarrow \infty}(1-r / x)=1
$$

By (2.2) and (2.3) can get:

$$
\pi(x)-\pi(x-r) \sim x \sum_{x-r \leq p \leq x} \frac{1}{p},
$$

According to Euler's asymptotic Formula (1.2), get

$$
\begin{aligned}
& \sum_{x \rightarrow r \leq p \leq x} \frac{1}{p} \sim \log \log x-\log \log (x-r)=\log \frac{\log x}{\log (x-r)} \\
= & \log \frac{\log x}{\log (x(1-r / x))}=\log \frac{\log x}{\log x+\log (1-r / x)},
\end{aligned}
$$

From this, we get 


$$
\sum_{x-r \leq p \leq x} \frac{1}{p} \sim \log \frac{\log x}{\log x-\left(\frac{1}{x / r}+\frac{1}{2(x / r)^{2}}+\frac{1}{3(x / r)^{3}}+\cdots+\frac{1}{k(x / r)^{k}}\right)},
$$

Take main item $\frac{1}{x / r}$, get:

$$
\begin{aligned}
& \sum_{x-r \leq p \leq x} \frac{1}{p} \sim \log \frac{\log x}{\log x-\frac{1}{x / r}}=\log \frac{1}{1-\frac{1}{(x / r) \log x}}=\log \left(1-\frac{1}{(x / r) \log x}\right)^{-1} \\
& =\frac{1}{(x / r) \log x}+\frac{1}{2(x / r)^{2}(\log x)^{2}}+\frac{1}{3(x / r)^{3}(\log x)^{3}}+\cdots+\frac{1}{k(x / r)^{k}(\log x)^{k}},
\end{aligned}
$$

Take the main item again $\frac{1}{(x / r) \log x}$, From this, we get

$$
\sum_{x \rightarrow r \leq p \leq x} \frac{1}{p} \sim \frac{r}{x \log x}
$$

By (2.5) Substituting (2.4),

$$
\pi(x)-\pi(x-r) \sim \frac{r}{\log x},(x \rightarrow \infty),
$$

This proves (1.3).

Now, let's look at the mean of the prime $p$, by $(x-r) \leq p \leq x$ can get

$$
p \approx \frac{(x-r)+x}{2}=x-r / 2,
$$

According to (2.4), we change $\mathrm{x}$ into an average $(x-r / 2)$ get

$$
\pi(x)-\pi(x-r) \sim(x-r / 2) \sum_{x-r \leq p \leq x} \frac{1}{p},
$$

For example $x=271, r=34,237 \leq p \leq 271$, prime number 241, 251, 257, 263, By (2.6) get

$$
\pi(271)-\pi(237) \sim 254(1 / 241+1 / 251+1 / 257+1 / 263)=4.02,
$$

actual $\pi(271)-\pi(237)=4$.

We divide $x$ into $\mathrm{n}$ parts, $n$ is a positive integer.

Let $x=2 n, r=2$, can get $(x-r / 2)=(2 n-1)$, by (2.6) we get

$$
\pi(2 n)-\pi(2 n-2)=(2 n-1) \sum_{2 n-2 \leq p \leq 2 n} \frac{1}{p},
$$

Therefore, we can improve the Riemann ladder function.

\section{Improved Riemann Ladder Function}

In 1859, German mathematician Riemann published a formula [2]:

$$
\pi(x)=\sum_{n=1}^{k} \frac{\mu(n) J\left(x^{1 / n}\right)}{n},
$$

Here (3.1) is Riemann's prime distribution formula [4]. Where $J\left(x^{1 / n}\right)$ is called 
Riemannian ladder function [7]. The calculation is very complicated.

According to the definition of Riemann ladder function:

$$
J(x)=\pi(x)+\frac{\pi\left(x^{1 / 2}\right)}{2}+\frac{\pi\left(x^{1 / 3}\right)}{3}+\cdots+\frac{\pi\left(x^{1 / k}\right)}{k}, k=\frac{\log x}{\log 2},
$$

A new ladder function is obtained by improving the ladder function

$$
J(x)=\sum_{0<p \leq 2} \frac{1}{p}+3 \sum_{2<p \leq 4} \frac{1}{p}+5 \sum_{4<p \leq 6} \frac{1}{p}+\cdots+p \sum_{2 n-2<p \leq 2 n} \frac{1}{p},
$$

Let $x \geq 2$, Integer $n$, prime $p$, get [4]

$$
\begin{gathered}
\pi(x)=J(x)+1 / 2, \\
J(x)=\sum_{n=1}^{a}(2 n-1) \sum_{2 n-2<p \leq 2 n} \frac{1}{p}, a=x / 2,
\end{gathered}
$$

Here (3.2) is an improved Riemannian prime distribution formula. It is strictly equal to $\pi(x)$.

For example $x=16, a=16 / 2=8, n=1,2,3,4,5,6,7,8$.

According to (3.2), a prime number is crossed each time, and the calculation is as follows:

$$
\pi(16)=1 \times \frac{1}{2}+3 \times \frac{1}{3}+5 \times \frac{1}{5}+7 \times \frac{1}{7}+11 \times \frac{1}{11}+13 \times \frac{1}{12}+\frac{1}{2}=6,
$$

In fact, $\pi(16)=6$, it is now prove that (3.2).

\section{Prove}

If there is a prime $p$ between $2 n-2$ and $2 n$, it must be

$$
p=2 n-1 \text {, }
$$

Set up $n=1,2,3, \cdots, a$, by (3.2) and (3.3) get,

$$
J(x)+\frac{1}{2}=\sum_{0<p \leq 2} \frac{1}{p}+3 \sum_{2<p \leq 4} \frac{1}{p}+5 \sum_{4<p \leq 6} \frac{1}{p}+\cdots+p \sum_{2 a-2<p \leq 2 a} \frac{1}{p}+\frac{1}{2},
$$

According to (3.4), we can get the

$$
J(x)+\frac{1}{2}=1 \times \frac{1}{2}+3 \times \frac{1}{3}+5 \times \frac{1}{5}+\cdots+p \times \frac{1}{p}+\frac{1}{2}=\pi(x),
$$

By (3.5) get

$$
\pi(x)=\sum_{n=1}^{a}(2 n-1) \sum_{2 n-2<p \leq 2 n} \frac{1}{p}+\frac{1}{2},
$$

Confirm (3.2) certification.

\section{Merdens' Theorem}

In 1874, the mathematician Merdens proved that [7]:

$$
\lim _{x \rightarrow \infty} \sum_{p \leq x} \frac{1}{p}-\log \log x=M,
$$

Here (4.1) is called: merdens theorem [8]. Where the mertensian constant 


$$
M=0.2614972128476427837554268386086958 \cdots,
$$

Let the coefficient $c(x)$ be obtained from (4.1)

$$
\sum_{p \leq x} \frac{1}{p}-\log \log x=c(x),
$$

For the convenience of using (4.2), we transform (3.2).

For example $x=8, a=8 / 2=4$, by (3.2) can get

$$
J(8)=\sum_{0<p \leq 2} \frac{1}{p}+3 \sum_{2<p \leq 4} \frac{1}{p}+5 \sum_{4<p \leq 6} \frac{1}{p}+7 \sum_{6<p \leq 8} \frac{1}{p},
$$

Among

$$
\begin{aligned}
& \sum_{0<p \leq 2} \frac{1}{p}=\sum_{p \leq 2} \frac{1}{p}, \\
& 3 \sum_{2<p \leq 4} \frac{1}{p}=3 \sum_{p \leq 4} \frac{1}{p}-3 \sum_{p \leq 2} \frac{1}{p}, \\
& 5 \sum_{4<p \leq 6} \frac{1}{p}=5 \sum_{p \leq 6} \frac{1}{p}-5 \sum_{p \leq 4} \frac{1}{p}, \\
& 7 \sum_{6<p \leq 8} \frac{1}{p}=7 \sum_{p \leq 8} \frac{1}{p}-7 \sum_{p \leq 6} \frac{1}{p},
\end{aligned}
$$

From the above

$$
\begin{aligned}
J(8) & =\sum_{p \leq 2} \frac{1}{p}+3 \sum_{p \leq 4} \frac{1}{p}-3 \sum_{p \leq 2} \frac{1}{p}+5 \sum_{p \leq 6} \frac{1}{p}-5 \sum_{p \leq 4} \frac{1}{p}+7 \sum_{p \leq 8} \frac{1}{p}-7 \sum_{p \leq 6} \frac{1}{p} \\
& =7 \sum_{p \leq 8} \frac{1}{p}-2 \sum_{p \leq 2} \frac{1}{p}-2 \sum_{p \leq 4} \frac{1}{p}-2 \sum_{p \leq 6} \frac{1}{p} \\
& =7 \sum_{p \leq 8} \frac{1}{p}-2 \sum_{p \leq 2} \frac{1}{p}-2 \sum_{p \leq 4} \frac{1}{p}-2 \sum_{p \leq 6} \frac{1}{p}-2 \sum_{p \leq 8} \frac{1}{p}+2 \sum_{p \leq 8} \frac{1}{p} \\
& =9 \sum_{p \leq 8} \frac{1}{p}-2\left(\sum_{p \leq 2} \frac{1}{p}+\sum_{p \leq 4} \frac{1}{p}+\sum_{p \leq 6} \frac{1}{p}+\sum_{p \leq 8} \frac{1}{p}\right),
\end{aligned}
$$

Generally speaking,

$$
J(x)=(x+1) \sum_{p \leq x} \frac{1}{p}-2\left(\sum_{p \leq 2} \frac{1}{p}+\sum_{p \leq 4} \frac{1}{p}+\sum_{p \leq 6} \frac{1}{p}+\cdots+\sum_{p \leq 2 a} \frac{1}{p}\right), a=x / 2,
$$

Replace (4.3) with (4.2), where

$$
\sum_{p \leq x} \frac{1}{p}=\log \log x+c(x),
$$

And

$$
\begin{aligned}
& \sum_{p \leq 2} \frac{1}{p}+\sum_{p \leq 4} \frac{1}{p}+\sum_{p \leq 6} \frac{1}{p}+\cdots+\sum_{p \leq 2 a} \frac{1}{p} \\
& =\log \log 2+c(2)+\log \log 4+c(4)+\log \log 6+c(6)+\cdots+\log \log x+c(x) \\
& =\sum_{n=1}^{a} \log \log (2 n)+\sum_{n=1}^{a} c(2 n),
\end{aligned}
$$

It is obtained by substituting (4.3) above 


$$
J(x)=(x+1)(\log \log x+c(x))-2 \sum_{n=1}^{a} \log \log (2 n)-2 \sum_{n=1}^{a}(2 n),
$$

Namely

$$
J(x)=(x+1) \log \log x-2 \sum_{n=1}^{a} \log \log (2 n)+(x+1) c(x)-2 \sum_{n=1}^{a}(2 n),
$$

Let $a=x / 2, s(x)$ denote the logarithmic part, which is obtained by substituting (3.2) above

$$
\pi(x)=s(x)+(x+1) c(x)-2 \sum_{n=1}^{x / 2} c(2 n)+\frac{1}{2}
$$

Among

$$
s(x)=(x+1) \log \log x-2 \sum_{n=1}^{x / 2} \log \log (2 n),
$$

Here (4.6) is strictly equal to $\pi(x)$.

For example $x=8, x / 2=4$, by (4.6) get

$$
\begin{aligned}
\pi(8)= & 9 \log \log 8-2 \sum_{n=1}^{4} \log \log (2 n)+9 c(8)-2 \sum_{n=1}^{4} c(2 n)+\frac{1}{2} \\
= & 9 \log \log 8-2(\log \log 2+\log \log 4+\log \log 6+\log \log 8) \\
& +9 c(8)-2(c(2)+c(4)+c(6)+c(8))+0.5,
\end{aligned}
$$

By (4.2) calculation coefficient

$$
\begin{aligned}
& c(2)=\sum_{p \leq 2}(1 / p)-\log \log 2=0.8665129 \ldots \\
& \left.c(4)=\sum_{p \leq 4}(1 / p)\right)-\log \log 4=0.50669907 \ldots \\
& \left.c(6)=\sum_{p \leq 6}(1 / p)\right)-\log \log 6=0.45013525 \ldots \\
& c(8)=\sum_{p \leq 8}(1 / p)-\log \log 8=0.4440911 \cdots
\end{aligned}
$$

Get

$$
\begin{aligned}
\pi(8)= & 9(0.73209937)-2(1.275418)+9(0.4440911) \\
& -2(0.8665129+0.50669907+0.4501352+0.4440911) \\
= & 4.000005=4,
\end{aligned}
$$

Actual $\pi(8)=4$.

By (4.6) we can prove the prime theorem with remainder.

\section{Prime Theorem with Remainder}

The remainder estimation of prime number theorem is very complicated. The key is to use the mertensian constant to calculate the coefficient.

Let's look at the remainder of (4.6). Among

$$
2 \sum_{n=1}^{x / 2} c(2 n)
$$

Let even numbers $y, x>y$, according to (3.2) $a=x / 2$, can get,

$$
x / 2=y / 2+(x-y) / 2,
$$


From this we get

$$
2 \sum_{n=1}^{x / 2} c(2 n)=2 \sum_{n=1}^{y / 2} c(2 n)+2 \sum_{n=1}^{(x-y) / 2} c(y+2 n)
$$

Substituting (4.6),

$$
\pi(x)=s(x)+(x+1) c(x)-2 \sum_{n=1}^{(x-y) / 2} c(y+2 n)+2 \sum_{n=1}^{y / 2} c(2 n)+\frac{1}{2},
$$

where $s(x)$ is the main term followed by the remainder. Now let's look at the remainder of (5.1). Among

$$
(x+1) c(x)-2 \sum_{n=1}^{(x-y) / 2} c(y+2 n)
$$

Here (5.2) is the key remainder. Let's see $c(x)$ and $c(y+2 n)$.

In order to let $c(y+2 n)$ approach $M$, let $y=2\left[x^{1 / 2}\right]$. According to (4.1) and (4.2), $x$ tends to infinity, and we obtain

$$
\begin{aligned}
& c(x) \rightarrow M \\
& c(y+2 n) \rightarrow M,
\end{aligned}
$$

By (5.2) get

$$
\begin{aligned}
& (x+1) c(x)-2 \sum_{n=1}^{(x-y) / 2} c(y+2 n) \rightarrow(x+1) M-2 \sum_{n=1}^{(x-y) / 2} M \\
& =(x+1) M-(x-y) M,
\end{aligned}
$$

Namely

$$
(x+1) c(x)-2 \sum_{n=1}^{(x-y) / 2} c(y+2 n) \rightarrow(y+1) M
$$

Let's look at a very small $x$.

For example $x=16, y=4,(x-y) / 2=6$, Substitute (5.3) and calculate according to (4.2)

$$
\begin{aligned}
& (16+1) c(16)-2 \sum_{n=1}^{6} c(4+2 n) \\
& =(16+1) c(16)-2(c(6)+c(8)+c(10)+c(12)+c(14)+c(16)) \\
& =17 \times 0.3242412-2(0.45013+0.44409+0.342158 \\
& \quad+0.3568+0.373601+0.3242) \\
& =5.5121-2(2.2911)=0.929918,
\end{aligned}
$$

By (5.3) get

$$
(y+1) M=5 \times 0.2614972 \cdots \approx 1.3074861,
$$

1.3074861 near 0.929918 .

$x$ The bigger, the closer $(y+1) M$.

Now, let's look at the scope of the remainder.

By (5.3) Substitute (5.1),

$$
\pi(x) \rightarrow s(x)+(y+1) M-2 \sum_{n=1}^{y / 2} c(2 n)+\frac{1}{2},
$$


For the $y$ of sufficient size, it is obvious that the estimation is

$$
M<(y+1) M<y \log x,
$$

Substitute (5.4) get

$$
\begin{aligned}
& \pi(x)>s(x)+M-2 \sum_{n=1}^{y / 2} c(2 n), \\
& \pi(x)<s(x)+y \log x-2 \sum_{n=1}^{y / 2} c(2 n),
\end{aligned}
$$

Let's look at the coefficient,

$$
2 \sum_{n=1}^{y / 2} c(2 n)=2 c(2)+2 c(4)+2 c(6)+\cdots+2 c(y),
$$

From (4.1) and (4.2) Confirm $M / 2<c(2 n)<1$, obviously

$$
2 \sum_{n=1}^{y / 2} \frac{M}{2}<2 \sum_{n=1}^{y / 2} c(2 n)<2 \sum_{n=1}^{y / 2} 1
$$

Get

$$
\frac{y M}{2}<2 \sum_{n=1}^{y / 2} c(2 n)<y
$$

By (5.6) Substitute (5.5) get

$$
\begin{aligned}
& \pi(x)>s(x)+M-y>s(x)-y \log x, \\
& \pi(x)<s(x)+y \log x-\frac{y M}{2}<s(x)+y \log x,
\end{aligned}
$$

Let $y=2\left[x^{1 / 2}\right]$, by (5.7) can get

$$
\pi(x)=s(x)+O\left(x^{1 / 2} \log x\right),
$$

By (5.8) Confirm, The prime theorem (1.6) is proved.

\section{Conclusions}

Previously, we discussed the Euler asymptotic formula

$$
\sum_{p \leq x} \frac{1}{p} \sim \log \log x .
$$

From (6.2), we prove the prime theorem in a simple way

$$
\pi(x) \sim r \sum_{n=1}^{x / r} \frac{1}{\log (n r)},
$$

Here (6.2) is a prime theorem without remainder.

We also discuss the distribution formula of prime number of Riemann

$$
\pi(x)=\sum_{n=1}^{a} \frac{\mu(n) J\left(x^{1 / n}\right)}{n},
$$

We make a great improvement on the distribution formula of Riemannian prime numbers

$$
\pi(x)=\sum_{n=1}^{a}(2 n-1) \sum_{2 n-2<p \leq 2 n} \frac{1}{p}+\frac{1}{2},
$$


This is a function strictly equal to $\pi(x)$. In this way, we prove that Riemann's conjecture is correct in principle.

According to the (6.3) theorem, we prove the strong prime number theorem

$$
\pi(x)=s(x)+O\left(x^{1 / 2} \log x\right),
$$

Here (6.6) is a prime theorem with remainder. Where $s(x)$ and $L i x$ are equal.

\section{Conflicts of Interest}

The author declares no conflicts of interest regarding the publication of this paper.

\section{References}

[1] Atiyah, M. and Singer, I.M. (1963) The Index of Elliptic Operators on Compact Manifolds. Bulletin of the American Mathematical Society, 69, 422-433. https://doi.org/10.1090/S0002-9904-1963-10957-X

[2] Ge, L.M. (2019) On the Riemann Zeta Function, I: KS-Transform. Acta Mathematica Sinica, Chinese Series, 62, 674.

[3] Lu, C.H. (2004) The Riemann Hypothesis (In Chinese). Tsinghua University Press, Beijing, Vol. 7, 9-18.

[4] Liu, J.F. (2013) Riemann Hypothesis Elementary Discussion. Progress in Applied Mathematics, 6, 74 .

[5] Li, X. (1997) The Positivity of a Sequence of Numbers and the Riemann Hypothesis. Journal of Number Theory, 65, 325-333. https://doi.org/10.1006/jnth.1997.2137

[6] Pan, C.D. and Pan, C.B. (1988) The Elementary Proof of Prime Number Theorem (In Chinese). Shanghai Science and Technology Press, Shanghai, 41-43.

[7] Rudin, W. (1987) Real and Complex Analysis, 3rd Edition, McGraw-Hill, New York.

[8] Riemann, B. (1859) Uber die Anzahl der Primzahlen unter Einer Gegebenen Grösse. Monatsber, Berlin Akad. 